\title{
Multimorbidität und Multi-Target-Therapie in der Phytotherapie
}

\author{
Saller, R ; Melzer, J
}

DOI: https://doi.org/10.1159/000351724

Posted at the Zurich Open Repository and Archive, University of Zurich ZORA URL: https://doi.org/10.5167/uzh-80242

Journal Article

Published Version

Originally published at:

Saller, R; Melzer, J (2013). Multimorbidität und Multi-Target-Therapie in der Phytotherapie. Forschende Komplementärmedizin, 20(Suppl 2):1.

DOI: https://doi.org/10.1159/000351724 


\title{
Multimorbidität und Multi-Target-Therapie in der Phytotherapie
}

\author{
Reinhard Saller ${ }^{\mathrm{a}}$ Jörg Melzer ${ }^{\mathrm{a}, \mathrm{b}, \mathrm{c}}$ \\ a Institut für Naturheilkunde, Universitätsspital Zürich, Schweiz \\ ${ }^{\mathrm{b}}$ Abteilung für Psychiatrie, Psychotherapie und Psychosomatik, Evangelisches Krankenhaus Königin-Elisabeth-Herzberge, \\ ${ }^{\mathrm{c}}$ Hochschule für Gesundheit und Sport, Berlin, Deutschland
}

Pflanzliche Arzneimittel sind strukturell und funktionell genuine Vielstoffgemische [1,2]. Diese Tatsache bedingt wissenschaftliche, aber auch praktisch-therapeutische Konsequenzen. Der Wirkstoff (das Vielstoffgemisch) muss z.B. über die konventionelle Inhaltsstoffanalyse hinaus auch funktionell charakterisiert werden; die reine Analytik der Einzelkomponenten oder die Annahme, Phytotherapeutika seien nur einfache Ansammlungen von Monosubstanzen, ergeben kein hinreichendes Funktionsbild. Die Einzelkomponenten gehen untereinander vielfältige strukturgebende und funktionell-plastische, zumeist schwache Bindungen ein. Das Geflecht der Interaktionen wirkt mit seinen vielfältigen Bindungsmöglichkeiten nach außen als flexibles Netzwerk auf die zahlreichen Targets des Organismus ein (Netzwerkpharmakologie) [3].

\section{Phytotherapeutika als Polypharmaka}

Der Wirkungsmechanismus setzt sich aus mehreren, zum Teil unabhängigen Einzelaktivitäten zusammen. Ein solches Arzneimittel wird eher multifokal, polypharmakologisch (Multi-Target-Eigenschaften mit/ohne Pleiotropie) und zumeist nicht selektiv wirken (breite biologische Aktivität). Dies kann erhebliche Vorteile bieten, unter anderem da viele Krankheiten auf dem komplexen Zusammenspiel zahlreicher Targets beruhen $[1,3]$. Multi-Target- und Pleiotropie-Eigenschaften können sich gerade bei Multimorbidität (komplexe Pathophysiologien) als günstig erweisen. Phytotherapeutika können Polypharmakotherapien vereinfachen («weakly ac- tive compounds, which attack various cellular targets» anstelle von «highly active compounds with a great selectivity» [4]). Wenn notwendig, lassen sich nichtselektive Multi-Targetmit selektiven Mono-Target-Wirkstoffen kombinieren.

\section{Phytotherapeutika als systemische Therapie}

Phytotherapeutische Mono- und Kombinationspräparate besitzen neben den indikationsbezogenen Wirkungen auch Gruppeneffekte, z.B. anti-inflammatorisch als «Systemeigenschaft» [1], zusätzlich zu beschwerdeorientierten Wirkungen, z.B. antidepressiv oder tonisierend [5]. Nicht jeder anti-inflammatorisch zu behandelnde Patient benötigt daher jeweils ein separat anti-entzündliches Phytotherapeutikum.

Häufig sind Phytotherapeutika als «multi-therapeutic drugs» nicht in allen potenziellen Anwendungsgebieten mit klinischen Studien geprüft $[1,4]$. Sie lassen sich aber durchaus versuchsweise in Situationen außerhalb der zugelassenen Indikationen anwenden, da notwendige Daten zu Qualität und Sicherheit bereits aus registrierten Anwendungsgebieten existieren. So können neu entdeckte, potenziell nützliche experimentelle Untersuchungsergebnisse der bereits lange verwendeten pflanzlichen Arzneimittel aufgegriffen werden. Diese translationale Vorgehensweise fällt in den Bereich einer Art reverser Pharmakologie [6]

In diesem Kontext gilt es, das Potenzial Tibetischer Arzneimittel als Prototypen von Netzwerkarzneien [7] auszuloten und ihren Einsatz bei komplexen Krankheitsbildern zu evaluieren.

\section{Literatur}

1 Saller R, Rostock M: Multimorbidität und MultiTarget-Therapie in der Phytotherapie. Praxis 2012; 101:1637-1642.

2 Wink M: Evolutionary advantage and molecula modes of action of multi-component mixtures used in phytomedicine. Curr Drug Metab 2008;9:996-1009.

3 Gertsch J: Botanical drugs, synergy, and network pharmacology: forth and back to intelligent mixtures. Planta Med 2011;77:1086-1098.
4 Azmi AS, Mohammad RM, Sarkar FH: Can network pharmacology rescue neutraceutical cancer research? Drug Discov Today 2012;17:807-809.

5 Saller R, Melzer J, Reichling J: Johanniskraut (Hypericum perforatum): ein plurivalenter Rohstoff für traditionelle und moderne Therapien. Forsch Komplementarmed Klass Naturheilkd 2003;10(suppl 1): 33-40.
6 Patwardhan B, Vaidya AD: Natural products drug discovery: accelerating the clinical candidate development using reverse pharmacology approaches. Indian J Exp Biol 2010;48:220-227.

7 Schwabl H, Vennos C, Saller R: Tibetische Rezepturen als pleiotrope Signaturen - Einsatz von Netzwerkarzneien bei Multimorbidität. Forsch Komplementmed 2013;20(suppl 2):35-40.

\section{KARGER}

Fax +497614520714

Information@Karger.com

www.karger.com
(C) 2013 S. Karger GmbH, Freibur

661-4119/13/0208-0001\$38.00/0

Accessible online at:

www.karger.com/fok
Prof. Dr. med. Reinhard Salle

Institut für Naturheilkunde

Universitätsspital Zürich

Rämistrasse 100, Postfach 131, 8091 Zürich, Schweiz

reinhard.saller@usz.ch 